\title{
Meningiomas del surco olfatorio. Tratamiento microquirúrgico radical por vía bifrontal
}

\author{
J.M. González-Darder; J.V Pesudo-Martínez*; V. Bordes-García; V. Quilis-Quesada; F. Talamantes-Escrivá;
} P. González-López y G. Masbout-Kayal

Servicios de Neurocirugía. Hospital Clínico Universitario de Valencia y *Hospital General de Castellón. Conselleria de Sanitat. Generalitat Valenciana.

Resumen

Objetivos. Describir los detalles técnicos del abordaje bifrontal para el tratamiento microquirúrgico radical de los meningiomas del surco olfatorio. Revisar los factores diagnósticos a valorar en la selección del abordaje quirúrgico de estos tumores.

Material y métodos. Se revisa una serie microquirúrgica de 35 tumores del surco olfatorio intervenidos por vía bifrontal.

Resultados. El volumen medio de las lesiones era de $85 \mathrm{cc}(4.4 \mathrm{~cm}$ de diámetro). El $65.7 \%$ presentaban edema cerebral perilesional relevante, el $80 \%$ hiperostosis en la base de implantación y el $\mathbf{2 8 . 6 \%}$ invasión de los senos paranasales. En todos los casos se realizó una exéresis grado 1 de Simpson. Hubo un éxitus por neumonía. La estancia hospitalaria fue de 3-20 días, con un Glasgow Outcome Scale 4-5 al alta en todos los casos y seguimiento medio de 55.2 meses. Como complicaciones relevantes destacan rinolicuorrea transitoria en dos casos e hidrocefalia en otro caso. Se ha identificado una recidiva local asintomática en un paciente a los 4 años de la cirugía.

Conclusiones. En nuestra experiencia la vía bifrontal ha permitido la exéresis radical de los meningiomas de suco olfatorio de gran tamaño. La disección microquirúrgica de los segmentos $\mathrm{A} 2$ de las arterias cerebrales anteriores es posible gracias a la existencia de un plano aracnoideo entre ellas y el tumor. La vascularización del tumor se controla abordando precozmente el tumor por la base y no se precisa embolización preoperatoria. La vía bifrontal permite un tratamiento agresivo de la hiperostosis, infiltración ósea e invasión de los senos paranasales. La reconstrucción de la fosa anterior se consigue con un colgajo perióstico pediculado.

PALABRAS CLAVE: Cirugía de base de cráneo. Colgajo perióstico pediculado. Craneotomía bifrontal. Meningioma del surco olfatorio.

Recibido: 21-06-10. Aceptado: 9-07-10
Olfactory groove meningiomas. Radical microsurgical treatment through the bifrontal approach

Objectives. To describe the microsurgical technique for the radical removal of olfactory groove meningiomas through the bifrontal approach. To review the diagnostic elements to be taken into account in the selection of the surgical approach to these tumours.

Materials and methods. A microsurgical series of $\mathbf{3 5}$ olfactory groove meningiomas operated on through a bifrontal craniotomy is reviewed.

Results. The mean tumoral volume was $85 \mathrm{cc}(4.4 \mathrm{~cm}$ diameter). A relevant peritumoral brain edema was found in $65.7 \%$ of cases, hyperostosis in the implantation base in $80 \%$ and paranasal sinus invasion in $28.6 \%$. A Sipmson grade 1 resection was achieved in every case. A patient died due to a postoperative pneumonia. Postoperative hospitalization time was between 3 and 20 days and at discharge all patients had a Glasgow Outcome Scale grade 4-5. The mean follow-up was $\mathbf{5 5 . 2}$ months. Two patients had postoperative transient rhinolicuorrhea and an additional patient developed hydrocephalus. An asymptomatic recurrence have been identified in a patient four years after surgery.

Conclusions. In our experience the bifrontal approach allowed the radical removal of huge olfactory groove meningiomas. The microdissection of the anterior cerebral artery $\mathbf{A 2}$ segments is possible thanks to the arachnoidal plane between vessels and tumor. Tumoral blood flow is secured by the early approaching of the base of the tumor and preoperative embolization is not necessary. Bifrontal approach allows an aggressive treatment of the hyperostosis, bone infiltration and paranasal sinus invasion. Anterior fossa reconstruction is done using a vascularized periosteal flap.

KEY WORDS: Bifrontal craniotomy. Olfactory groove meningiomas. Skull base surgery. Vascularized periosteal flap. 


\section{Introducción}

Los meningiomas del surco olfatorio suponen menos del 5\% de los meningiomas de la base del cráneo. Cuando se diagnostican se trata habitualmente de lesiones de gran tamaño y rodeadas de edema perilesional en un alto porcentaje de casos. A pesar de ser una lesión bien conocida y relativamente frecuente, todavía existe discusión acerca de cual es la vía de abordaje más útil para su manejo microquirúrgico ${ }^{1-9,11-14,16,17}$. Los objetivos de este trabajo han sido la revisión de la experiencia propia en el tratamiento microquirúrgico radical de los meningiomas del surco olfatorio a través de una vía de abordaje bifrontal, estudiar los factores que influyen en la toma de decisión de la vía de abordaje y, como consecuencia de ello y de la revisión de la literatura más reciente, hacer algunas recomendaciones sobre la aplicación de procesos de mejora continua para alcanzar la excelencia en el tratamiento de estas complejas lesiones.

\section{Material y métodos}

En el trabajo se incluyen una serie de meningiomas del surco olfatorio intervenidos mediante abordaje bifrontal por el primer autor entre los años 1996 y 2009. Para ello, se han revisado retrospectivamente las historias clínicas y los estudios de imagen, obteniendo datos epidemiológicos, clínicos, morfológicos y evolutivos relevantes.

\section{Técnica quirúrgica}

El paciente se coloca en decúbito supino con la cabeza elevada y en discreta extensión. Se practica una incisión bicoronal obteniendo un amplio colgajo perióstico pediculado sobre el reborde orbitario y con la vascularización procedente de ambas arterias supraorbitarias. Posteriormente se realiza una craneotomía bifrontal lo más basal posible, se liga el seno longitudinal y se secciona el falx separando ambos lóbulos frontales para exponer el tumor. En los tumores muy grandes se hace una osteotomía de las apófisis orbitaria de ambos frontales, techo de órbita y nasion para extirpar en bloque la barra orbitaria. Tras separar los lóbulos frontales se fresa la crista galli y se coagulan las arterias que salen por su base. En general, es posible retraer el tumor coagulando su cápsula y separarlo de la duramadre de la fosa anterior, aunque pronto debe abrirse para realizar la descompresión interna con aspirador ultrasónico, lo que permite ir despegándolo más y más de la base. Esto se aprovecha para coagular las adherencias aracnoideas y pequeños vasos aferentes. La maniobra se repite una y otra vez, buscando intracapsularmente el auténtico implante del tumor, que se reconoce por la convergencia de los vasos y la trama fibrosa. Todas estas maniobras van devascularizando el tumor que, una vez ahuecado, se movilizada tirando de la cápsula. Un detalle de interés es que no se debe empujar el tumor hacia atrás por el peligro de estirar los nervios ópticos todavía no liberados, por lo que la cápsula se tracciona hacia delante, colocando lentinas entre ella y la base del lóbulo frontal. Esta maniobra se facilita coagulando la cápsula para retraerla. La coagulación y sección de todas las ramas arteriales aracnoideas devasculariza adicionalmente el tumor y la de las gruesas venas capsulares impide sangrados a distancia por su ruptura al movilizar la cápsula. En los tumores grandes suele ser ahora el momento de resecar las porciones más anteriores de la cápsula y de progresar con la coagulación de las aferencias vasculares basales, aunque siempre debemos dejar suficiente cápsula para mantener un buen plano entre el tumor y los lóbulos frontales.

En este momento conviene dedicar la atención a encontrar los segmentos A2 de las arterias cerebrales anteriores. En este punto es importante la información proporcionada por las RNM de las relaciones entre el tumor y las A2. Sin embargo, las arterias fronto-polares y fronto-orbitarias no se identifican en la RNM y suelen tener íntimas relaciones y emitir ramas hacia el tumor. Consecuentemente, la progresión entre el tumor y los lóbulos frontales debe ser cuidadosa, ya que al separar ambos elementos debemos identificar inicialmente las arterias fronto-polares y frontoorbitarias y seguirlas hasta su salida del segmento A2. Las ramas de la $\mathrm{A} 2$ pueden ser coaguladas y seccionadas en este punto en caso de que entren en el tumor. Por el contrario, los segmentos A2 deben ser separados del tumor, aunque se encuentre rodeados del mismo, ya que su mayor tamaño les permite mantener una doble capa aracnoidea que las aísla del tumor. En todo caso, el segmento A2 debe ser respetado aún a costa de dejar tumor a su alrededor. Una separación violenta del tumor y los lóbulos frontales puede producir una hemorragia incontrolable o una isquemia con graves secuelas si no se toman las precauciones indicadas.

Liberadas las A2 se ha entrado en la cisterna de la lamina terminalis y se procede a identificar el quiasma óptico. Se pueden ahora resecar grandes fragmentos de cápsula y tumor exponiendo el ala del esfenoides en ambos lados y toda la fosa anterior hasta el planum esfenoidal, de donde se desinserta el tumor y se coagulan provisionalmente las aferencias basales. En este momento pueden quedar mamelones tumorales que deben ser separados cuidadosamente de ambos nervios ópticos y tallo hipofisario o que se insinúan en las cisternas carotídeas.

Finalizada la exéresis tumoral se presta atención a la base de la fosa anterior. Inicialmente se extirpa de forma amplia la duramadre y luego se fresa el hueso hiperostósico hasta identificar la mucosa de las celdas etmoidales. Durante el fresado pueden reabrirse las aferencias arteriales, aunque una vez extirpado el tumor reducen apre- 
ciablemente su flujo de forma espontánea. Esta zona se recubre antes del cierre de la craneotomía con el colgajo perióstico pediculado preparado previamente, que puede suturarse a la duramadre del ala esfenoidal, y que también cubre y sella los senos frontales habitualmente abiertos en la craneotomía.

\section{Resultados}

La serie comprende 35 pacientes, 18 hombres y 17 mujeres, con edades comprendidas entre 41 y 87 años. El volumen tumoral se calculó midiendo los tres diámetros mayores y aplicando la fórmula (a.b.c)/2, resultando entre 15 y $220 \mathrm{cc}$, con una media de $85 \mathrm{cc}$, lo que corresponde a una esfera de $4.4 \mathrm{~cm}$ de diámetro. Había un edema perilesional relevante en $23(65.7 \%)$ casos, hiperostosis en la base de implante en $28(80 \%)$ casos e invasión de senos paranasales en 10 (28.6\%) casos. Todos los pacientes fueron estudiados mediante TAC y RNM, practicando un total de 8 estudios angiográficos sin ninguna embolización preoperatoria. En todos los casos se realizó una exéresis grado 1 de Simpson. La estancia hospitalaria fue de 320 días con un GOS al alta de 4-5 en 34 pacientes y un éxitus $(2.8 \%)$ por complicaciones respiratorias en el único paciente con recidiva tras una resección subtotal en otro centro y bajo Karnofsky. Como complicaciones relevantes destacan dos casos de rinolicuorrea transitoria y un caso de hidrocefalia. En el periodo de seguimiento (media 55.2 meses) sólo se ha identificado una recidiva asintomática a los 4 años de la cirugía.

\section{Discusión}

Los meningiomas son tumores habitualmente benignos histológicamente y que pueden ser curados con su exéresis. Por ello, el tratamiento de elección debe ser quirúrgico y encaminado a lograr una resección local radical grado 1 de Simpson. Los meningiomas de la base de cráneo como los de surco olfatorio presentan dificultades quirúrgicas adicionales, pero el planteamiento terapéutico debe ser el mismo.

\section{Aspectos anatomo-clínicos}

Los meningiomas del surco olfatorio agrupan un amplio número de lesiones que crecen alrededor de la línea media de la fosa anterior, desde la apófisis crista galli del etmoides a lo largo de la lámina cribiforme hasta la línea de sutura etmoidoesfenoidal, que la separa del planum esfenoidal ${ }^{15}$. A pesar de que suelen alcanzar gran tamaño y su aspecto es simétrico, su base de implantación puede ser relativamente pequeña o estar asentada paramedialmente, extendiéndose excéntricamente a lo largo de la duramadre de la fosa anterior. La base de implantación suele estar asociada a una hiperostosis reactiva a la invasión tumoral del hueso y pueden crecer en sentido basal invadiendo las celdas etmoidales. Las series quirúrgicas describen una presentación variable de ambos hechos, pero en la nuestra la hiperostosis alcanza el $80 \%$ de los casos y la invasión etmoidal casi un tercio de los casos. La superficie de contacto con los lóbulos frontales suele ser rugosa y desplazan los nervios olfatorios, ópticos y quiasma, manteniendo casi siempre íntegra la aracnoides de las cisternas de la lamina terminalis, quiasmática, carotídea y del ala del esfenoides. La vascularización de los meningiomas del surco olfatorio procede predominantemente de ramas etmoidales (anteriores y posteriores) de la arteria oftálmica y el drenaje venoso puede ser muy bizarro. Sin embargo, en la cirugía, se reconocen aferencias de pequeño calibre de las arterias cerebrales anteriores y de sus ramas fronto-polares y fronto-orbitarias. Ocasionalmente estos vasos pueden estar adheridos o rodeados por el tumor, aunque siempre existe una capa aracnoidea alrededor de las ramas de cierto calibre que permite su disección. La superficie del tumor esta recorrida por grandes venas de drenaje.

Aunque los meningiomas del surco olfatorio crecen de forma lenta y en una zona de escasa expresividad neurológica, lo cierto es que la presentación clínica que lleva al diagnóstico es muchas veces abrupta. Sólo entonces, retrospectivamente, se reconocen síntomas menores o de desarrollo paulatino que pasaron desapercibidos durante tiempo. Es llamativa la frecuente asociación de un gran edema cerebral peritumoral uni o bilateral que en nuestra serie aparece en los dos tercios de los pacientes y que suele relacionarse con un debut clínico abrupto. El diagnóstico se hace actualmente con la RNM, aunque el estudio preoperatorio con TAC es recomendable para conocer la disposición de los senos paranasales, erosión o hiperostosis inducidas por el tumor o calcificaciones intratumorales. Las series de RNM en T1 con gadolinio ofrecen una detallada información anatómica de la lesión, pero en las series T2 se identifica el edema cerebral peritumoral y es posible reconocer las relaciones de las arterias cerebrales anteriores con el tumor. Este último es un importante detalle en el manejo microquirúrgico del tumor y es valorado mucho mejor en la RNM que con la angiografía.

\section{Vías de abordaje}

Los meningiomas de surco olfatorio sintomáticos deben ser tratados quirúrgicamente con el objetivo de obtener una resección radical grado 1 de Simpson. La finalidad del tratamiento quirúrgico es la resección completa de la lesión mejorando la clínica y minimizando las posibilidades de complicaciones, secuelas y recidivas. Con esta finalidad se han usado diferentes abordajes ${ }^{1-5,7-9,11-14,16,17}$, que se pueden agrupar en unilaterales (subfrontal unilateral; pterional; 
Tabla 1

Meningiomas del surco olfatorio. Resultados de series quirúrgicas recientes

\begin{tabular}{|c|c|c|c|c|c|c|c|}
\hline autor & casos & $\begin{array}{l}\text { diámetro } \\
\text { medio } \\
\text { (cm) }\end{array}$ & $\begin{array}{l}\text { vías de } \\
\text { abordaje }\end{array}$ & $\begin{array}{c}\text { resección } \\
\text { total o } \\
\text { Simpson 1-2 } \\
\text { (\%) }\end{array}$ & $\begin{array}{c}\text { mortalidad } \\
(\%)\end{array}$ & $\begin{array}{l}\text { segui- } \\
\text { miento } \\
\text { medio } \\
\text { (meses) }\end{array}$ & $\begin{array}{l}\text { recurren- } \\
\text { cias (\%) }\end{array}$ \\
\hline $\begin{array}{l}\text { Aguiar, } \\
2009^{1}\end{array}$ & 21 & 4.3 & $\begin{array}{l}7 \text { bifrontal } \\
11 \text { pterional } \\
3 \text { fronto-orbital }\end{array}$ & g lobal 100 & 4.8 & - & 19 \\
\hline $\begin{array}{l}\text { Bassiouni } \\
2007^{2}\end{array}$ & 55 & - & $\begin{array}{l}36 \text { bifrontal } \\
13 \text { pterional } \\
\text { resto otras }\end{array}$ & g lobal 100 & 0 & 67.2 & 8.9 \\
\hline $\begin{array}{l}\text { Colli } \\
2007^{3}\end{array}$ & 17 & - & bifrontal & 94.1 & 11.8 & - & 0 \\
\hline $\begin{array}{l}\text { El-Bahy } \\
2009^{5}\end{array}$ & 18 & $\begin{array}{r}7<4 \\
11>4\end{array}$ & frontolateral & 77.8 & 5.6 & - & - \\
\hline $\begin{array}{l}\text { Nakamura } \\
2007^{11}\end{array}$ & 82 & $\begin{array}{l}4.89 \\
\text { bifrontal } \\
\quad 4.16 \\
\text { frontolateral }\end{array}$ & $\begin{array}{l}46 \text { bifrontal } \\
34 \text { frontolateral } \\
\text { otras resto }\end{array}$ & $\begin{array}{c}93.5 \\
91.2 \\
-\end{array}$ & $\begin{array}{l}8.7 \\
0\end{array}$ & 63.4 & 4.9 \\
\hline $\begin{array}{l}\text { Romani } \\
2009^{16}\end{array}$ & 66 & - & $\begin{array}{l}\text { lateral } \\
\text { supraorbital }\end{array}$ & 91 & 0 & 45 & 6 \\
\hline $\begin{array}{l}\text { Skeptor } \\
2005^{17}\end{array}$ & 80 & 4,6 & $\begin{array}{l}35 \text { bifrontal } \\
18 \text { subfrontal } \\
12 \text { subcraneal } \\
\text { resto otras }\end{array}$ & global 90 & 0 & 70.8 & 2.5 \\
\hline $\begin{array}{l}\text { González } \\
2010\end{array}$ & 35 & 4.4 & bifrontal & 100 & 2.8 & 55.2 & 2.8 \\
\hline
\end{tabular}

frontotemporal; orbitocigomático; supraorbitario) o bilaterales (subfrontal bilateral aislado o extendido con la resección de la barra orbitaria; transfrontal; abordajes craneofaciales). Más recientemente se han desarrollado vías de abordaje subcraneal o transbasal endoscópica ${ }^{6}$. En nuestra experiencia personal sólo un pequeño meningioma incidental fue tratado por vía supraciliar y algunas lesiones pequeñas fueron extirpadas por vía frontotemporal. En varios pacientes se extirparon meningiomas de surco olfatorio de tamaño pequeño en abordajes para meningiomas sintomáticos de otra localización y asequibles en el mismo acto quirúrgico. Sin embargo, la mayor parte de los tumores se extirparon por vía subfrontal bilateral o bifrontal, ya que eran de gran tamaño, y son los incluidos en este trabajo. En la tabla 1 se recogen los datos mas relevantes de algunas serie modernas donde se detallan los resultados obtenidos con las diferentes vías de abordaje. Aunque cada autor defiende los beneficios de una vía determinada, parece apreciarse que la vía bifrontal se prefiere para los tumores de mayor tamaño y que hay tendencia en las series más modernas a usar la vía subfrontal unilateral.

Aspectos a considerar en la selección del abordaje quirúrgico

Todo abordaje neuroquirúrgico debe ser individualizado 


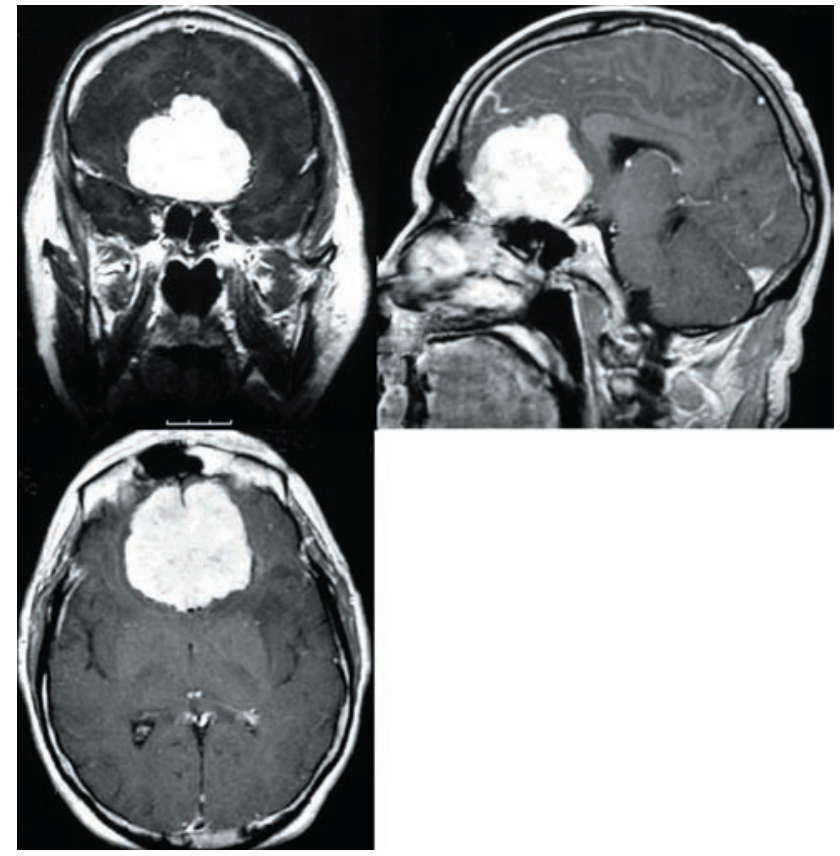

Figura 1. Aspecto típico del meningioma del surco olfatorio en un estudio de RNM-T1 con gadolinio en cortes axial, coronal y sagital. Hay un mínimo edema peritumoral, se aprecian las arterias A2 adosadas al tumor y existe una pequeña invasión de los senos etmoidales. El volumen tumoral calculado es de $69 \mathrm{cc}$.

y para los tumores del surco olfatorio se deben tener en cuenta los siguientes elementos: volumen tumoral determinado por el tamaño de la lesión y el edema peritumoral; implante tumoral e invasión de las estructuras de la base del cráneo; vascularización y relación tumor-vasos; minimizar las complicaciones y secuelas neurológicas.

Tamaño y volumen tumoral. Al diagnóstico, los meningiomas del surco olfatorio suelen ser lesiones de gran tamaño. En nuestra serie, el volumen medio era de $85 \mathrm{cc}$, lo que corresponde a una esfera de $4.4 \mathrm{~cm}$ de diámetro. En las series quirúrgicas, el tamaño de corte para ser considerados grandes es de $3 \mathrm{~cm}$ de diámetro $\mathrm{y}$, en este caso, se recomienda de forma generalizada el abordaje bifrontal, incluso con resección adicional de la barra orbitaria para minimizar la retracción frontal ${ }^{3}$. En los últimos años, se recomienda por algunos autores la vía subfrontal para minimizar el abordaje e incluso algunos autores como Reisch y Perneczky han defendido abordajes mínimamente invasivos ${ }^{14}$. El edema peritumoral es un factor adicional en la toma de decisiones. El mecanismo fisiopatológico del edema cerebral en los meningiomas es discutido, pero se considera vasogénico y se relaciona con una gran cantidad de factores, aunque se desconoce su causa exacta. El edema condiciona un efecto de masa adicional, ya que aumenta el contenido intracraneal de forma rápida y puede precipi-

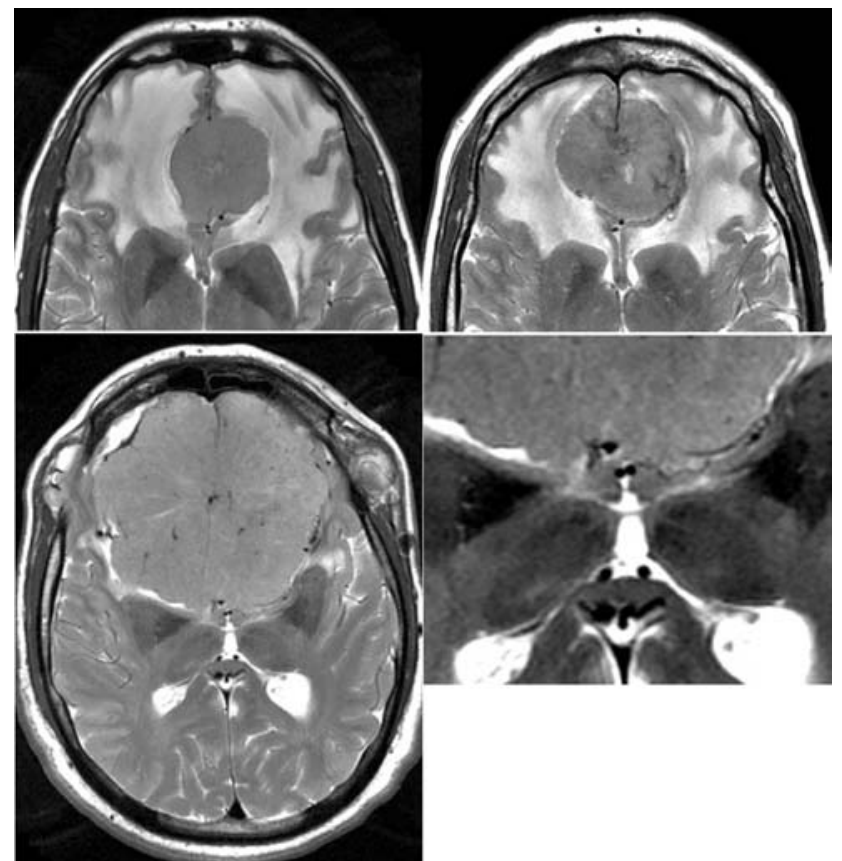

Figura 2. Relaciones vasculares de los meningiomas del surco olfatorio con el segmento A2 de la arteria cerebral anterior estudiados con RNM-T2. El los dos casos de la fila superior existe un gran edema cerebral asociado de ambos lóbulos frontales y las arterias aparecen libres. En el caso inferior no hay edema cerebral y las arterias aparecen invaginadas en el seno del tumor. En todos los casos las arterias pudieron ser separadas del tumor.

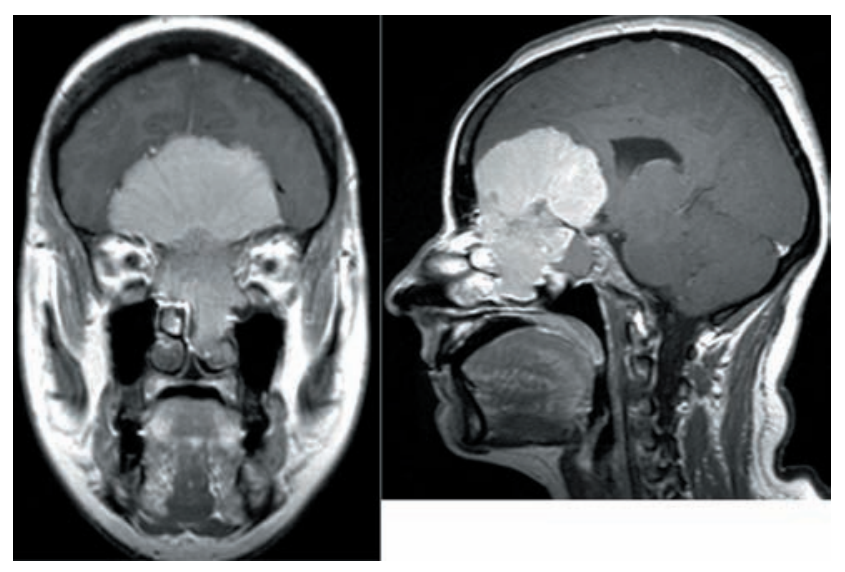

Figura 3. Meningioma de surco olfatorio con invasión masiva de senos etmoidales, fosas nasales y órbita izquierda. Hay ocupación del seno esfenoidal. Estudio de RNM-T1 con gadolinio. El tumor fue completamente resecado con osteotomía de la barra orbitaria y la fosa anterior fue reparada con un colgajo perióstico pediculado obtenido en la craneotomía. 
tar la clínica y aun teniendo buena respuesta clínica a los corticoides no muestra cambios evidentes en la imagen. En todo caso, un abordaje amplio como el bifrontal sería beneficioso en estos casos.

Implante tumoral e invasión local. El implante tumoral está en la línea media de la fosa anterior y, aunque aquí la duramadre y el hueso subyacente son delgados, el tumor suele crecer de forma exofítica. De forma sistemática se evidencia crecimiento tumoral en la dura adyacente, pero es excepcional el crecimiento en placa. Con frecuencia variable se aprecia hiperostosis reactiva a la infiltración tumoral del hueso y con frecuencia existe una auténtica invasión de los senos etmoidales del tumor, siendo excepcional la invasión orbitaria ${ }^{8,11}$. El objetivo de la cirugía debe ser una resección radical o grado 1 de Simpson, lo que en los meningiomas del surco olfatorio implica la resección del tumor, de la duramadre de la fosa anterior, hueso hiperostósico infiltrado y senos paranasales eventualmente invadidos, lo que obliga al fresado del hueso hasta la mucosa de los senos paranasales etmoidales, ya que está bien demostrada la relación entre la recidiva del meningioma benigno y el grado de resección ${ }^{8,11,12}$. De todas formas, la frecuencia de recurrencia global es relativamente baja en la literatura (0-41\% / 3.7-25 años / series macro y microquirúrgicas $)^{8}$. La recurrencia es nula si el seguimiento es corto, pero aumenta con el tiempo de observación (5-41\% a partir de los 10 años), lo que puede llevar a la conclusión de plantear un tratamiento local menos agresivo en pacientes ancianos. También Lagares et $\mathrm{al}^{9}$ son poco partidarios de una actuación local agresiva y, probablemente influidos por su nula tasa de recidivas tras un seguimiento medio de 6.2 años en una serie de 27 casos sin invasión ósea, proponen que sería suficiente una resección grado 2 de Simpson. Frente a ello, la recomendación general que compartimos es la resección radical Simpson grado 1 con reconstrucción de la base de la fosa anterior con un colgajo perióstico obtenido antes de la craneotomía y, en caso de recidivas sin colgajo disponible, usando fascia lata para evitar plastias sintéticas o heterólogas $^{12}$. La experiencia demuestra que los senos paranasales y la base del cráneo son los lugares de predilección de las recurrencias y esto es atribuible a la incompleta resección del hueso invadido y al recrecimiento en los bordes del resección ${ }^{12}$.

Vascularización. El aporte vascular de los meningiomas del surco olfatorio procede esencialmente de las ramas etmoidales anteriores y posteriores de la arteria oftálmica. Estas aferencias pueden obliterarse precozmente siguiendo las pautas de abordaje descritas por nosotros. Excepcionalmente pueden excluirse identificando dichas ramas en un abordaje intraorbitario periorbitario, ya que las arterias etmoidales anteriores se localizan a $24 \mathrm{~mm}$ y las posteriores a $36 \mathrm{~mm}$ de la cresta anterior del surco nasolacrimal, pudiendo ser identificadas y coaguladas de forma selectiva antes del abordaje directo al tumor ${ }^{10,18}$. El resto de los vasos proceden de las arterias fronto-polares y fronto-orbitarias, ramas del segmento A2 de la arteria cerebral anterior, que son coaguladas al resecar la cápsula. Las propias A2 pueden estar envueltas y, excepcionalmente, la adventicia invadida por el tumor. En nuestra experiencia la angiografía no aporta datos relevantes para el tratamiento, siendo suficiente la secuencia T2 de la RNM que demuestra las relaciones entre el tumor y los segmentos A2. Consecuentemente, somos de la opinión de que la embolización preoperatoria no tiene ningún valor en el tratamiento de estos tumores e incluso puede ser peligrosa al existir peligro de ceguera ${ }^{10,18}$.

Minimizar complicaciones y secuelas. Las complicaciones tras la cirugía de los meningiomas de surco olfatorio se relacionan con empeoramiento de los síntomas preoperatorios, problemas locales (infección, edema intra o postoperatorio, hematoma y fístula de liquido cefalorraquídeo) o sistémicos (neumonía). La mortalidad postoperatoria varía entre el $0-11 \%$ según las series (tabla 1 ). El-Bahy ${ }^{5}$ describe un éxitus debido a la lesión de ambas cerebrales anteriores envueltas por el tumor. El éxitus de nuestra serie se debió a una neumonía en un paciente con hipertensión endocraneal severa y bajo Karnofski por recidiva masiva. Sin embargo, la mayor parte de las series muestran mejoría de los síntomas preoperatorios ${ }^{7}$ y en tumores de pequeño tamaño es posible incluso plantearse la conservación de uno o ambos nervios olfatorios.

\section{Resección radical por vía bifrontal}

Los detalles de la técnica quirúrgica del abordaje bifrontal se encuentran descritos en la literatura ${ }^{8,11,13,15} \mathrm{y}$ las particularidades de nuestro abordaje se han detallado en este trabajo, en especial la disección del segmento A2 de ambas cerebrales anteriores. Sin embargo, cualquier abordaje quirúrgico debe acoplarse a las necesidades de cada paciente. El abordaje bifrontal es muy versátil, ya que puede ampliarse con la resección de la barra orbitaria ${ }^{3}$ y permitir un acceso prácticamente ilimitado a los senos paranasales, fosas nasales y clivus para resecar a cualquier extensión craneobasal del tumor ${ }^{15}$, siendo la reconstrucción de la base del cráneo muy segura con el colgajo perióstico pediculado ${ }^{12}$. Por ello, el abordaje bifrontal debe ser considerado como primera opción para los tumores de gran tamaño ( $>4 \mathrm{~cm}$ de diámetro), con los segmentos $\mathrm{A} 1$ o A2 de las arterias cerebrales anteriores invaginados es su espesor en el estudio de RNM con secuencia T2, si hay invasión de los senos paranasales o necesidad de reconstrucción de la fosa anterior. Algunos puntos débiles del abordaje bifrontal incluyen la necesidad de abrir los senos frontales, ligar el seno longitudinal y manipular ambos lóbulos frontales. En nuestra opinión, la exéresis radical de los meningiomas de 
surco olfatorio de gran tamaño es una tarea formidable, en donde hay que intercalar a lo largo de la misma una detallada atención a varios aspectos muy concretos y exigentes que condicionan el éxito global del tratamiento, pero el abordaje bifrontal permite un buen control de todos ellos.

\section{Bibliografía}

1. Aguiar, P.H., Tahara, A., Almeida, A.N., Simm, R., Silva, A.N., Maldaun, M.V., Panagopoulos, A.T., Zicarelli, C.A., Silva, P.G.: Olfactory groove meningiomas: approaches and complications. J Clin Neurosci 2009; 16: 1168-1173.

2. Bassiouni, H., Asgari, S., Stolke, D.: Olfactory groove meningiomas: functional outcome in a series treated microsurgically. Acta Neurochir (Wien) 2007; 149: 109-121.

3. Chi, J.H., Parsa, A.T., Berger, M.S., Kunwar, S., McDermott, M.W.: Extended bifrontal craniotormy for midline anterior fossa meningiomas: minimization of retraction-related edema and surgical outcomes. Neurosurgery 2006; 59 (ONS Suppl 4): ONS426-ONS434.

4. Colli, B.O., Carlotti, C.G. Jr, Assirati, J.A. Jr, Santos, M.B,. Neder, L., Santos, A.C., Batagini, N.C.: Olfactory groove meningiomas: surgical technique and follow-up review. Arq Neuropsiquiat. 2007 ; 65: 795-799.

5. El-Bahy, K.: Validity of the frontolateral approach as a minimally invasive corridor for olfactory groove meningiomas. Acta Neurochir (Wien) 2009; 151: 11971205.

6. Gardner, P.A., Kassam, A.D., Thomas, A., Snyderman, C.H., Carrau, R.L., Mintz, A.R., Prevedello, D.M.: Endoscopic endonasal resection of anterior cranial base meningiomas. Neurosurgery 2008; 63: 36-54.

7. Gazzeri, R., Galarza, M, Gazzeri, G.: Giant olfactory groove meningioma: ophthalmological and cognitive outcome after bifrontal microsurgical approach. Acta Neurochir (Wien) 2008; 150: 1117-1125.

8. Hentschel, S.J., DeMonte, F.: Olfactory groove meniniomas. Neurosurg Focus 2003; 14: article 4.

9. Lagares, A., Lobato, R.D., Castro, S., Alday, R., De la Lama, A., Alén, J.F., González, P.: Meningiomas del surco olfatorio: Revisión de una serie de 27 casos. Neurocirugía 2001; 12: 17-22.

10. McDermott, M.W., Rootman, J., Durity, F.: Subperios- teal, subperiorbital dissection and division of the anterior and posterior ethmoid artery for meniniomas of the cribiform plate and planum sphenoidale. Technical note. Neurosurgery 1995; 36: 1215-1219.

11. Nakamura, M., Struck, M., Roser, F., Vorkapic, P., Samii, M.: Olfactory groove meningiomas: clinical outcome and recurrence rates after tumor removal through the frontolateral and bifrontal approach. Neurosurgery 2006; 60: 844-852.

12. Obeid, F., Al-Mefty, O.: Recurrence of olfactory groove meningiomas. Neurosurgery 2003; 53: 534-543.

13. Ojemann, R.G.: Olfactory groove meningiomas. En Al-Mefty, O. (ed): Meningiomas. Raven Press Ltd, New York, 1991 pp 383-393.

14. Reisch, R., Perneczky, A.: Ten-year experience with the supraorbital subfrontal approach through an eyebrow skin incision. Neurosurgery 2005; 57 (ONS Suppl 3): 242-255.

15. Rhoton, A.L.: The anterior and middle cranial base. Neurosurgery 2002; 51 (suppl 1): 273-302.

16. Romani, R., Lehecka, M., Gaal, E., Toninelli, S., Celik, O., Niemelä, M., Porras, M., Jääskeläinen, J., Hernesniemi, J.: Lateral supraorbital approach applied to olfactory groove meningiomas: experience with 66 consecutive patients. Neurosurgery 2009; $65: 39-52$.

17. Spektor, S., Valarezo, J., Fliss, D.M., Gil, Z., Cohen, J., Goldman, J., Umansky, F.: Olfactory groove meningiomas from neurosurgical and ear, nose, and throat perspectives: approaches, techniques, and outcomes. Neurosurgery. 2005; 57 (4 Suppl): 268-280.

18. White, D.V., Sincoff, E.H., Abdulrauf, S.I.: Anterior ethmoidal artery: microsurgical anatomy and technical considerations. Neurosurgery 2005; 56 (2 Suppl): 406-10.

González-Darder, J.M.; Pesudo-Martínez, J.V.; BordesGarcía, V.; Quilis-Quesada, V.; Talamantes-Escrivá, F.; González-López, P.; Masbout-Kayal, G.: Meningiomas del surco olfatorio. Tratamiento microquirúrgico radical por vía bifrontal. Neurocirugía 2011; 22: 133-139.

Correspondencia: José M. González Darder. Servicio de Neurocirugía. Hospital Clínico Universitario. Avda. Blasco Ibáñez, 17. 46010-Valencia.

gonzalez_jos@gva.es 